\title{
Towards the Quark-Gluon Plasma
}

\author{
Peter Braun-Munzinger \\ Gesellschaft für Schwerionenforschung \\ 64220 Darmstadt, Germany
}

We discuss recent experimental results in the field of ultra-relativistic nuclear collisions. The emerging "picture" is a collectively expanding, initially hot and dense fireball in which strangeness- and low-mass di-lepton pair production are enhanced and $\mathrm{J} / \Psi$ production is suppressed compared to expectations from nucleon-nucleon collisions. It is argued that, taken together, these data provide circumstantial evidence that a (at least partly) partonic phase was produced in such collisions.

\section{Introduction}

Research with ultra-relativistic nuclear collisions aims at producing, in the laboratory, quark-gluon plasma. This new state of matter is predicted to exist at high temperatures and/or high baryon densities. Specifically, numerical solution of QCD using lattice techniques imply that the critical temperature (at zero baryon density) is about $170 \mathrm{MeV}$ [1]. Comprehensive surveys of the various experimental approaches of how to produce such matter in nucleus-nucleus collisions have been given recently [2 4]. Here we focus on a few very selected aspects, namely radial flow, strangeness production, dilepton production, and $\mathrm{J} \Psi$ measurements and explore possible correlations among these observations. The aim is to elucidate possible connections of these observations to the QCD phase transition.

\section{Radial Flow}

A large body of data on transverse momentum (or transverse mass) distributions of hadrons demonstrates that the inverse slope constant of these (in general exponential) spectra scales linearly with particle mass $\mathrm{m}$. The experimental facts for $\mathrm{Pb}+\mathrm{Pb}$ collisions at SPS energy have been compiled recently [2] and are shown in Fig. 11. The observed linear relationship is naturally interpreted in terms of a collectively expanding fireball, where $p_{t}=p_{t}^{\text {thermal }}+m \gamma_{t} \beta_{t}$ [2,3] with $\beta_{t}$ the transverse flow velocity and $\gamma_{t}=\sqrt{1 /\left(1-\beta_{t}^{2}\right)}$. Using the known relationship between $\left\langle p_{t}\right\rangle$ and T,m one may deduce a mean value of the transverse velocity of $\left\langle\beta_{t}>\approx 0.4\right.$, in good agreement with results from hydrodynamic descriptions [3]. However, the T-m relation shown in Fig. 1 also implies $\mathrm{T}=180 \mathrm{MeV}$ for $\mathrm{m}=0$, not consistent with the current interpretation that thermal freeze-out takes place at around $\mathrm{T}=120 \mathrm{MeV}$ [3].

The now detailed measurements of transverse momentum distributions also imply little centrality dependence of the observed slope constants, in conflict with an interpretation 
of these observations in terms of initial state scattering [3].

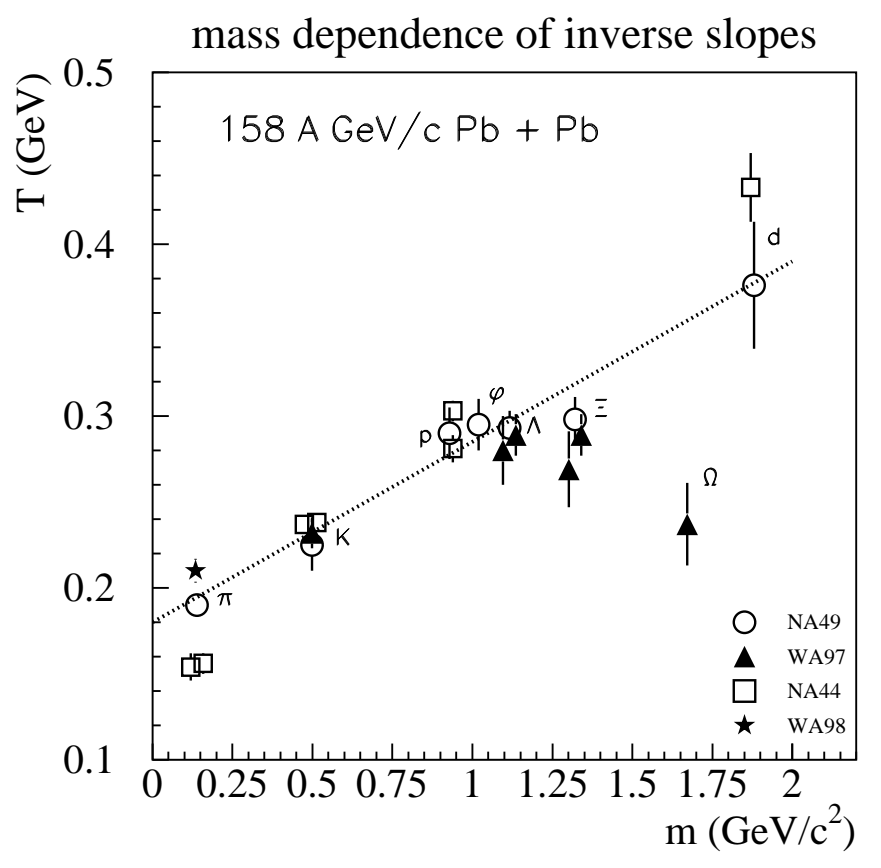

Figure 1. Inverse slope parameters vs particle mass.

An interesting and somewhat puzzling deviation from the general trend is observed for multi-strange baryons: the corresponding slope parameters are significantly smaller than expected. This has been interpreted as evidence for early freeze-out [5]. It could be due to very small cross sections for elastic pion-strange baryon scattering.

The overall conclusion, however, is that the picture of a collectively expanding fireball has survived all tests of the past years.

\section{Strangeness Enhancement and Equilibration}

The production yields of strange hadrons are significantly increased in ultra-relativistic nuclear collisions compared to what is expected from a superposition of nucleon-nucleon collisions. This has been observed by several experiments both at the AGS and at the SPS. To demonstrate the degree of enhancement observed we show, in Fig. 2, the results of the WA97 collaboration for multi-strange baryons [6].

The observed enhancement of more than one order of magnitude can currently not be understood within any of the hadronic event generators ${ }^{\text {t }}$. Surprizingly, it is quantitatively explained if one assumes complete chemical equilibrium in the hadronic phase of the collision [7]. Similar observations have been made for analyses of S-induced SPS data [8] and for AGS data 9.

How chemical equilibration can be reached in a purely hadronic collision is not clear in view of the small production cross section for strange and especially multi-strange

\footnotetext{
${ }^{1}$ For a discussion see the proceedings of the Quark Matter 99 conference, Nucl. Phys. A, to be published.
} 


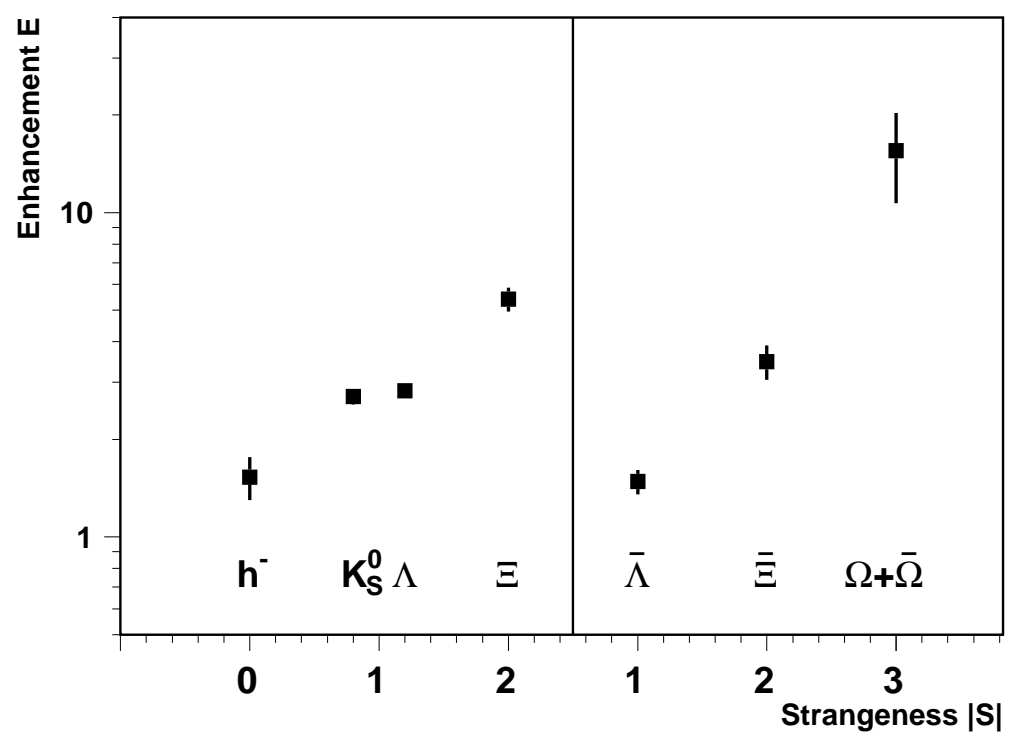

Figure 2. Strangeness enhancement in $\mathrm{Pb}+\mathrm{Pb}$ collisions for multi-strange baryons. The data are from the WA97 collaboration [6].

hadrons. In fact, system lifetimes of the order of $50 \mathrm{fm} / \mathrm{c}$ or more are needed for a hot hadronic system to reach full chemical equilibration [10]. Such lifetimes are at variance with lifetime values established from interferometry analyses, where upper limits of about $10 \mathrm{fm} / \mathrm{c}$ are deduced 11 .

Another very interesting observation is that the chemical potentials $\mu$ and temperatures T resulting from the thermal analyses of [7-9] place the systems at chemical freeze-out very close to where we currently believe is the phase boundary between plasma and hadrons.

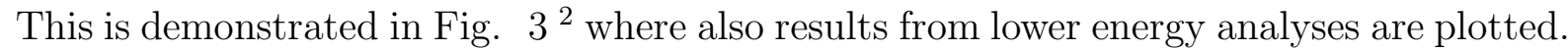
The freeze-out trajectory (solid curve through the data points) is just to guide the eye but follows closely the empirical curve of [12].

The closeness of the freeze-out parameters $(T, \mu)$ to the phase boundary might be the clue to the apparent chemical equilibration in the hadronic phase: if the system prior to reaching freeze-out was in the partonic (plasma) phase, then strangeness production is determined by larger partonic cross sections as well as by hadronization. Slow cooking in the hadronic phase is then not needed to produce the observed large abundances of strange hadrons. Early simulations of strangeness production in the plasma and during hadronization support this interpretation at least qualitatively [13]. Faced with the present results a new theoretical look seems mandatory.

\section{Enhancement of Low Mass Dilepton Pairs}

The CERES and HELIOS/3 collaborations found that, in central nucleus-nucleus collisions at SPS energy, low mass $(\mathrm{m}<800 \mathrm{MeV})$ dilepton pairs are produced at yields which are significantly larger than expected from nucleon-nucleon collisions [14 16 . The enhancement is concentrated at low pair transverse momentum [17 as can be seen from

\footnotetext{
${ }^{2}$ This is an updated version of the figure shown in [2, 3].
} 


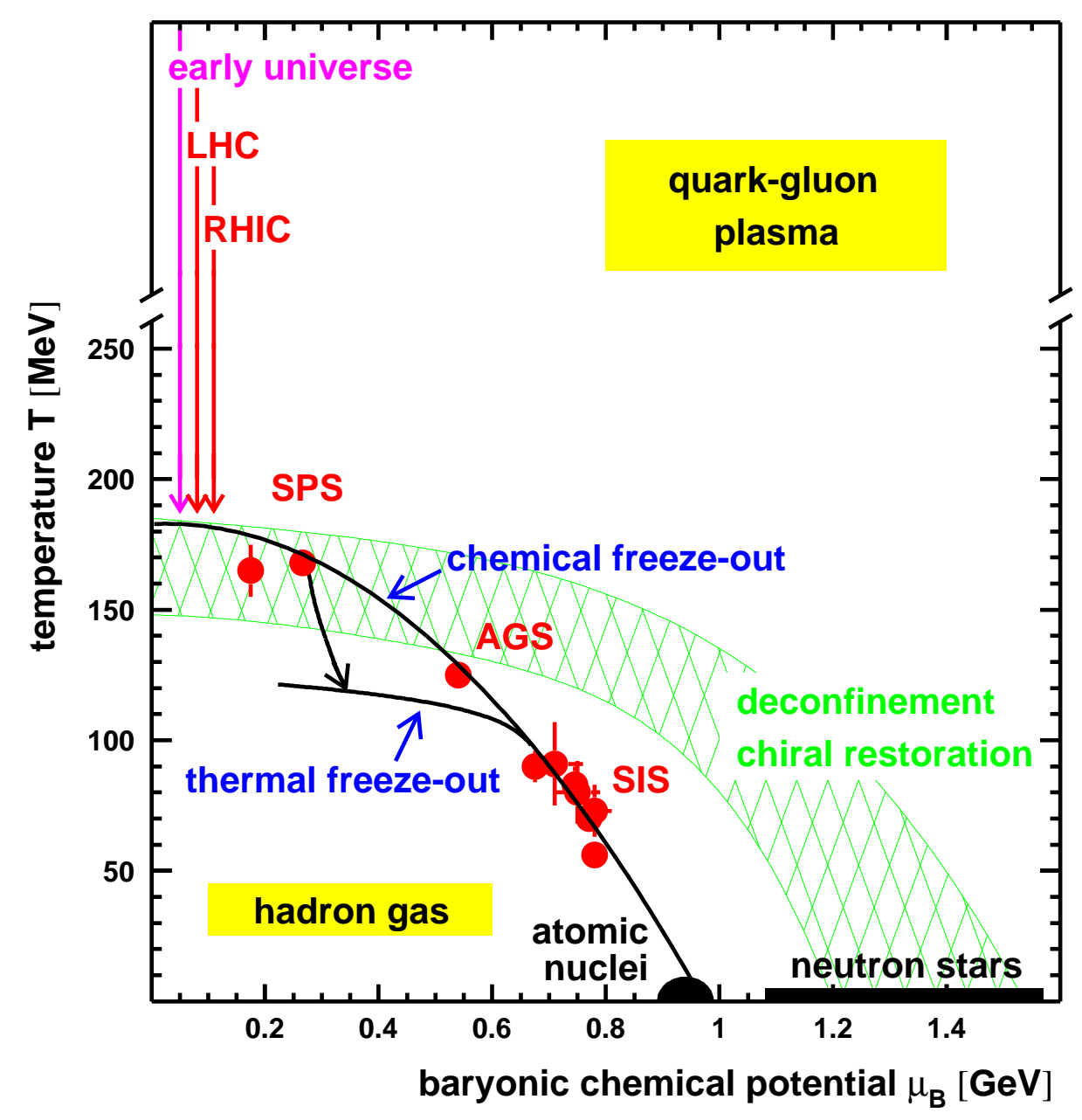

Figure 3. Phase diagram of hadronic and partonic matter. The hadrochemical freezeout points are determined from thermal model analyses of heavy ion collision data at SIS, AGS and SPS energy. The hatched region indicates the current expectation for the phase boundary based on lattice QCD calculations at $\mu=0$. The arrow from chemical to thermal freeze-out for the SPS corresponds to isentropic expansion.

the recent CERES data which are presented in Fig. 1.

The observed enhancement has been attributed to changes of the mass and/or width of the $\rho$ meson in the hot and dense fireball. The still somewhat controversial situation has been reviewed recently [19]. Here we want to add two points. First, the enhancement sets in at centralities corresponding to less than $35 \%$ of the total inelastic cross section [17]. This implies impact parameters $b<8 \mathrm{fm}$ (see Fig. 2 in [3]. From thereon it scales quadratically with particle multiplicity. Secondly, the $\rho$ mesons are formed according to Fig. 3 at around $\mathrm{T}=165 \mathrm{MeV}$. These two facts suggest that pion number (i.e. temperature) and $\pi-\pi$ collisions in the medium are at the origin of the observed enhancement. 


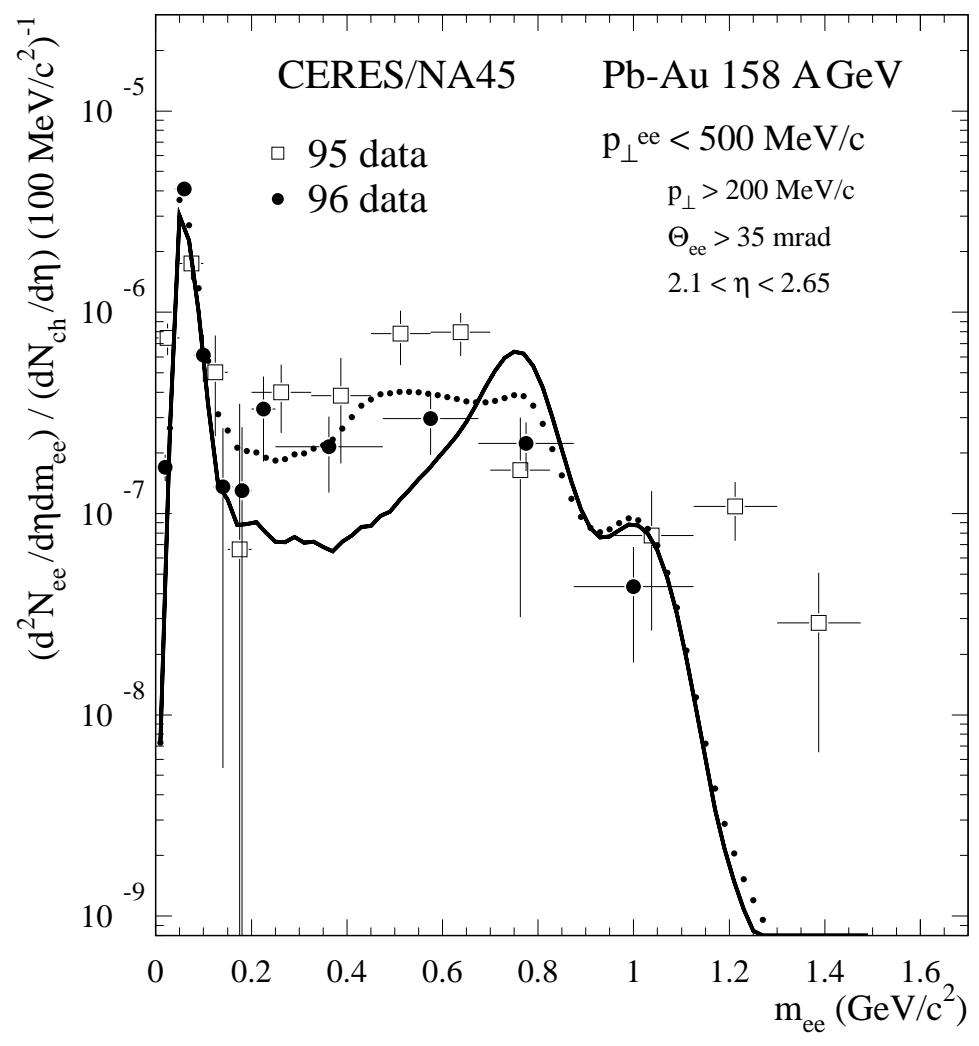

Figure 4. Invariant mass distribution of electron pairs for central $\mathrm{Pb}-\mathrm{Au}$ collisions and low pair transverse momentum. The solid line represents the expectation for the yield based on extrapolating nucleon-nucleon collision results. The dashed line is obtained by taking medium modifications of the $\rho$ meson into account 18

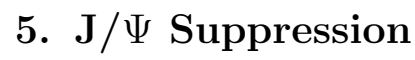

The suppression of $\mathrm{J} / \Psi$ mesons (compared to what is expected from hard scattering models) was early on predicted [20] to be a signature for color deconfinement. Data for Sinduced collisions exhibited a significant suppression but systematic studies soon revealed that such suppression exists already in p-nucleus collisions and is due to the absorption in (normal) nuclear matter of a color singlet $c \bar{c} g$ state that is formed on the way towards $\mathrm{J} / \Psi$ production. The situation has been summarized in [21,22,2].

The data for $\mathrm{Pb}+\mathrm{Pb}$ collisions now exhibit clear evidence for anomalous absorption beyond the standard absorption expected for such systems. The most recent results are summarized in Fig. 5, taken from [23,24]. There seems to be a break away from the standard absorption curve at around a transverse energy value of $40 \mathrm{GeV}$ corresponding to an impact parameter of about $8 \mathrm{fm} \mathrm{[23].} \mathrm{We} \mathrm{note} \mathrm{that} \mathrm{this} \mathrm{impact} \mathrm{parameter} \mathrm{value}$ agrees with the value from where on anomalous dilepton enhancement is observed by the

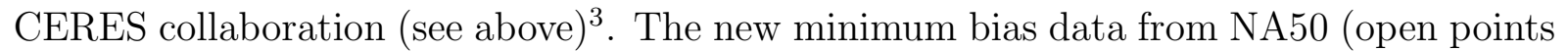
in Fig. 5) with their much smaller error bars very much accentuate the difference to the standard absorption curve.

Whether these data provide unambiguous evidence for the existence of a deconfined

\footnotetext{
${ }^{3}$ The data from CERES do not reach lower centralities corresponding to larger impact parameters. Hence this point is not yet "water-tight".
} 
phase in central $\mathrm{Pb}+\mathrm{Pb}$ collisions is hotly debated. However, there is at present no convincing explanation of the observed data in standard scenarios without plasma. The theoretical curves in Fig. [0 show this. All calculations from the Giessen group [25], from the Kahana team [26], from Capella's group [27], and from the Frankfurt group using UrQMD [28] are based on models for the destruction of charmonium by comoving pions, strings, etc. The corresponding dissociation cross sections are poorly known [29]. Despite very different assumptions about these cross sections and despite a number of other nontrivial assumptions (see, e.g. the discussion in [30]) none of the calculations reproduce the data. For example, comparing the Giessen calculation with the 1996 data (solid points in Fig. 5i) yields a reduced $\chi^{2}$ of larger than 4, while comparison of any of the calculations with the high statistics minimum bias data (open points in Fig. 5) yields reduced $\chi^{2}$ values of larger (sometimes much larger) than 10 . We conclude that the charmonium suppression observed by the NA50 collaboration in central $\mathrm{Pb}+\mathrm{Pb}$ collisions is highly non-trivial.

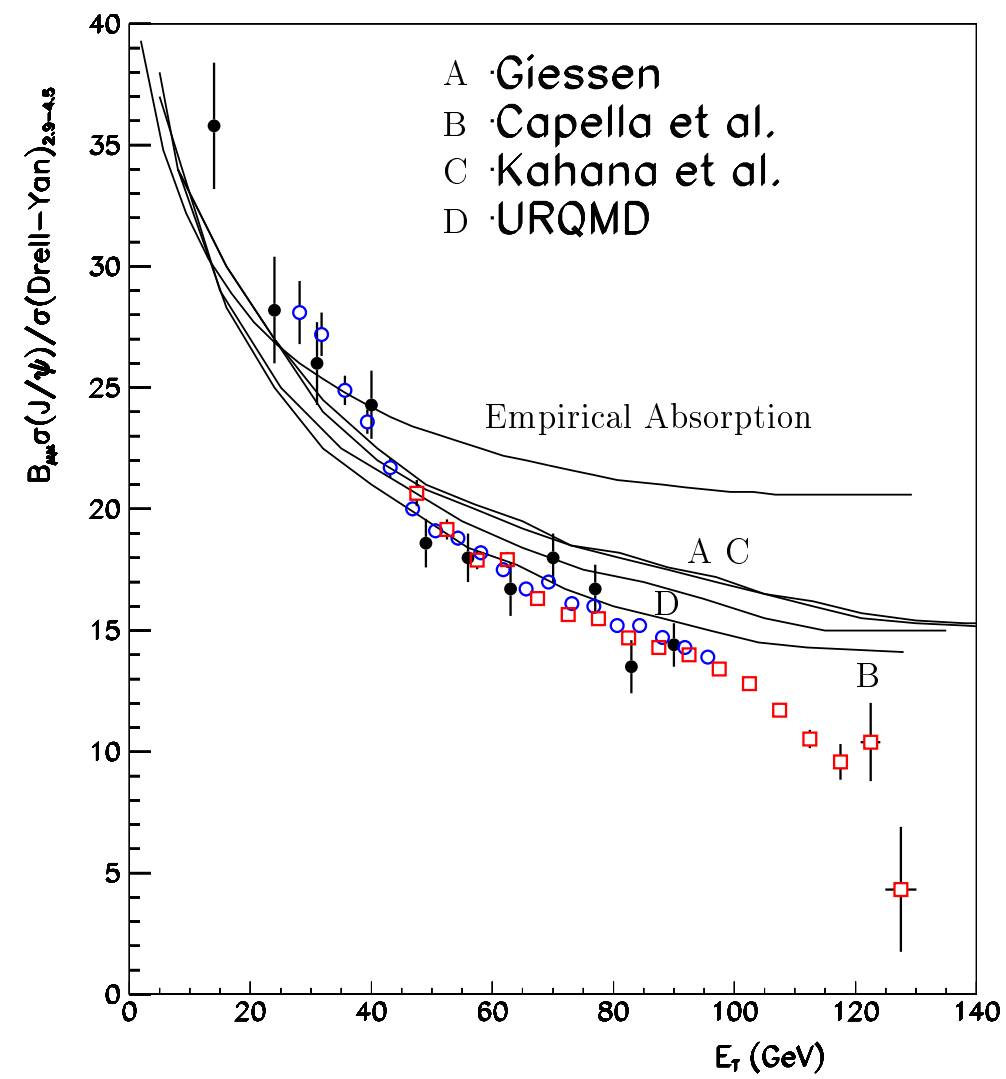

Figure 5. Transverse energy dependence of $\mathrm{J} / \Psi$ production in $\mathrm{Pb}+\mathrm{Pb}$ collisions normalized to the Drell-Yan cross section. Data and the empirical absorption curve are from the Na50 collaboration [23,24]. The various theoretical distributions summarize summarize attempts to describe the observations in "conventional" scenarios. For details see text. 


\section{Summary and Outlook}

Taken together, the observations of flow, strangeness enhancement, enhancement of lowmass dilepton pairs, and charmonium suppression lend strong support to the interpretation that, during the course of a central $\mathrm{Pb}+\mathrm{Pb}$ collision at SPS energy, an at least partly deconfined state of matter, i.e. of quark-gluon plasma, has been created. Two further rounds of experiments at the SPS will provide the possibility to consolidate this picture.

Meanwhile, experiments at the RHIC collider are about to commence and the planning for the ALICE experiment at the LHC is well underway. Physics prospects for experiments at these accelerators are bright 31,32]. Extrapolating from the results of the AGS and SPS program we expect much higher energy densities and temperatures at collider energy. Production and study of a deconfined phase over a large space-time volume should be possible.

\section{REFERENCES}

1. F. Karsch, Proc. Lattice99, Nucl. Phys. B (in print), hep-lat/9909006.

2. J. Stachel, Proc. INPC, Paris, August 1998, Nucl. Phys. A654 (1999) 119c, nuclex/9903007.

3. P. Braun-Munzinger and J. Stachel, Nucl. Phys. A638 (1998) 3c.

4. S. Bass, M. Gyulassy, H. Stöcker, W. Greiner, J.Phys. G25 (1999) R1-R57.

5. H. van Hecke, H. Sorge, N. Xu, Phys. Rev. Lett. 81 (1998) 5764.

6. E. Andersen et al., Wa97 Coll., J. Phys. G: Nucl. Part. Phys. 25 (1999) 171, E. Andersen, et al., WA97 Coll., Phys. Lett. B449 (1999) 401.

7. P. Braun-Munzinger, I. Heppe, J. Stachel, nucl-th/9903010, Phys. Lett B(in print).

8. P. Braun-Munzinger, J. Stachel, J. P. Wessels, N. Xu, Phys. Lett. B 365 (1996) 1.

9. P. Braun-Munzinger, J. Stachel, J. P. Wessels, N. Xu, Phys. Lett. B 344 (1995) 43.

10. J. Sollfrank and U. Heinz in: Quark Gluon Plasma 2, R.C. Hwa, editor, World Scientific 1996, p. 555.

11. H. Appelshäuser et al., NA49 collaboration, Eur. Phys. J. C2 (1998) 661.

12. J. Cleymans and K. Redlich, nucl-th/9903063.

13. H. W. Barz, B. L. Friman, J. Knoll, H. Schulz, Nucl. Phys. A519 (1990) 831.

14. G. Agakichiev et al., CERES collaboration, Phys. Rev. Lett. 75 (1995) 1272.

15. G. Agakichiev et al., CERES collaboration, Phys. Lett. B422 (1998) 405.

16. M. Masera for the HELIOS collaboration, Nucl. Phys. A590 (1995) 93c.

17. B. Lenkeit for the CERES collaboration, Proc. Quark Matter 99 conference, Torino, June 1999, Nucl. Phys. A(in print).

18. R. Rapp, proceedings Rencontres de Moriond '98, nucl-th/9804065; R. Rapp and C. Gale, hep-ph/9902268.

19. R. Rapp and J. Wambach, Adv. Nucl. Phys. (in print), nucl-th/9909229.

20. T. Matsui and H. Satz, Phys. Lett. B178 (1986) 416.

21. C. Lourenco, Nucl. Phys. A610 (1996) 552c.

22. D. Kharzeev, Nucl. Phys. A638 (1998) 279c.

23. M.C. Abreu et al., NA50 collaboration, Phys. Lett. B450 (1999) 456.

24. C. Cicalo for the NA50 collaboration, Proc. Quark Matter 99 conference, Torino, June 
1999, Nucl. Phys. A(in print).

25. J. Geiss, C. Greiner, E.L. Bratkovskaya, W. Cassing, U. Mosel, Phys. Lett. B447 (1999) 31; J. Geiss, C. Greiner, E.L. Bratkovskaya, W. Cassing, nucl-th/9810059.

26. D.E. Kahana, S.H. Kahana, Prog. Part. Nucl. Phys. 42 (1999) 395; D.E. Kahana, S.H. Kahana, nucl-th/9908063.

27. N. Armesto, A. Capella, E.G. Ferrero, Phys. Rev. C59 (1999) 395.

28. C. Spieles, R. Vogt, L. Gerland, S.A. Bass, M. Bleicher, H. Stöcker, W. Greiner, hep-ph/9902337.

29. P. Braun-Munzinger and K. Redlich, nucl-th/9908026.

30. B. Müller, Proc. Quark Matter 99 conference, Torino, June 1999, Nucl. Phys. A(in print), nucl-th/9906029.

31. P. Braun-Munzinger, Proc. Quark Matter 99 conference, Torino, June 1999, Nucl. Phys. A(in print), nucl-exp/9908007.

32. S. Bass et al., Proc. Quark Matter 99 conference, Torino, June 1999, Nucl. Phys. A(in print), nucl-th/9907090. 